\title{
Plasma Properties in the Plume of a Hall Thruster Cluster
}

\author{
Brian E. Beal* and Alec D. Gallimore ${ }^{\dagger}$ \\ University of Michigan, Ann Arbor, Michigan 48109 \\ and \\ James M. Haas ${ }^{\ddagger}$ and William A. Hargus Jr. ${ }^{\S}$ \\ Air Force Research Laboratory, Edwards Air Force Base, California 93524
}

\begin{abstract}
The Hall thruster cluster is an attractive propulsion approach for spacecraft requiring very high-power electric propulsion systems. Plasma density, electron temperature, and plasma potential data collected with a combination of triple langmuir probes and floating emissive probes in the plume of a low-power, four-engine Hall thruster cluster are presented. Simple analytical formulas are introduced that allow these quantities to be predicted downstream of a cluster based solely on the known plume properties of a single thruster.
\end{abstract}

\section{Nomenclature}

$A=$ area of one electrode

$A_{S}=$ surface area of sheath surrounding an electrode

$B=$ magnetic field strength

$E \quad=$ electric field strength

$e=$ electron charge

$k_{b}=$ Boltzmann's constant

$m_{e}=$ electron mass

$m_{i}=$ ion mass

$n=$ electron number density

$n_{0}=$ reference density

$T_{e}=$ electron temperature

$T_{e, 0}=$ reference electron temperature

$V_{d 2}=$ voltage measured between triple probe electrodes 1 and 2

$V_{d 3}=$ voltage applied between triple probe electrodes 1 and 3

$V_{f}=$ floating potential

$\gamma=$ ratio of specific heats

$\delta=$ sheath thickness

$\lambda_{D}=$ electron Debye length

$\phi \quad=$ plasma potential

$\phi_{T}=$ thermalized potential

Subscript

$j=$ contribution from an individual thruster

\section{Introduction}

$\mathbf{F}$ UTURE space missions will require electric propulsion systems capable of operating at very high-power levels compared to those currently in use. ${ }^{1,2}$ One method being considered for reaching these power levels involves clustering multiple devices of moderate

Received 11 July 2003; revision received 5 May 2004; accepted for publication 19 June 2004. Copyright (C) 2004 by the American Institute of Aeronautics and Astronautics, Inc. All rights reserved. Copies of this paper may be made for personal or internal use, on condition that the copier pay the $\$ 10.00$ per-copy fee to the Copyright Clearance Center, Inc., 222 Rosewood Drive, Danvers, MA 01923; include the code 0748-4658/04 \$10.00 in correspondence with the CCC.

*Doctoral Candidate, Plasmadynamics and Electric Propulsion Laboratory; currently Principal Development Engineer, Aerojet, Inc., P.O. Box 97009, Redmond, WA 98052; brian.beal@ rocket.com. Member AIAA.

${ }^{\dagger}$ Professor, Aerospace Engineering, Plasmadynamics and Electric Propulsion Laboratory, 1320 Beal Avenue. Associate Fellow AIAA.

${ }^{\ddagger}$ Research Scientist, Spacecraft Propulsion Branch, 1 Ara Road. Member AIAA.

${ }^{\S}$ Research Scientist, Spacecraft Propulsion Branch, 1 Ara Road. Senior Member AIAA. power to reach the total throughput desired. An attractive propulsion option for this class of mission is the Hall thruster due to its low specific mass, high thrust density, and high reliability. ${ }^{1,2}$ In an effort to understand the technical issues related to operating multiple Hall thrusters in close proximity to each other, a cluster of four Busek BHT-200-X3 200-W class devices has been studied. ${ }^{3-6}$

A cluster of thrusters may have a slightly lower efficiency and higher dry mass than a single, similarly powered thruster because larger engines have historically outperformed smaller thrusters. In contrast to this potential disadvantage in performance, however, recently published work has concluded that a cluster also offers several advantages over a monolithic thruster. ${ }^{1,2}$ Examples of the benefits of clustering include improved system reliability due to the inherent redundancy of running multiple engines and the ability to throttle the system by simply turning off one or more thrusters. ${ }^{1,2}$ Throttling the system in this way allows the cluster to operate at lower power without running any of the individual thrusters at off-design conditions. This characteristic of a cluster may prove beneficial on missions where either the available power or the propulsive needs change as a function of time. For example, a high-power cluster of Hall thrusters could be used for the initial low Earth orbit to geosynchronous Earth orbit transfer of a geosynchronous communications satellite. When its final destination is reached, one element of the cluster could then be used for north-south station keeping. A final advantage of clustering is the high degree of system scalability. In principle, once the technical issues involved with operating a cluster are fully understood, a single flight-qualified engine could support a wide range of missions requiring various power levels by simply clustering the appropriate number of thrusters. Thus, enhanced scalability and flexibility make clusters attractive for some missions despite the potential reduction in performance compared to large monolithic thrusters.

Although using a cluster of commercially available thrusters for primary propulsion appears to be advantageous for some missions, there are several systems integration issues that must be addressed before clusters can be used in flight. ${ }^{1,2}$ For example, it is imperative that the interaction of the plasma plumes both among the thrusters and with the spacecraft be understood. In an effort to address this issue, the electron number density, electron temperature, and plasma potential downstream of a low-power cluster were measured using a combination of electrostatic probes. In each case, the profiles recorded in the cluster plume were compared to those measured downstream of an individual thruster. These data have been used to develop methods for predicting the plume properties downstream of a cluster of thrusters based solely on measurements taken downstream of a single unit. As discussed in detail elsewhere, ${ }^{6}$ this ability is crucial to allow development of very high-power Hall thruster clusters without full-scale qualification tests because construction of ground-test facilities capable of supporting such systems is expected to be extremely expensive. 


\section{Experimental Apparatus}

Cluster

The cluster used in this experiment was composed of four Busek BHT-200-X3 200-W class Hall thrusters. An earlier version of this thruster was reported to operate at an anode efficiency of $42 \%$ and specific impulse of $1300 \mathrm{~s}$ while providing $12.4 \mathrm{mN}$ of thrust at the nominal operating conditions. ${ }^{7}$ Each thruster had a mean discharge channel diameter of $21 \mathrm{~mm}$ and was operated on xenon propellant. The thrusters were arranged in a $2 \times 2$ grid with approximately $11.4 \mathrm{~cm}$ between the centerlines of nearest neighbors. For the experiments discussed here, each thruster was coupled to its own hollow cathode whose keeper remained powered throughout the tests, although other configurations have been studied and are discussed elsewhere. ${ }^{6}$ Typical operating conditions for the BHT-200 are given in Table $1{ }^{3}$ Figure 1 shows the cluster during operation.

\section{Vacuum Facilities}

The electron number density, electron temperature, and plasma potential measurements presented in this paper were obtained in chamber 6 at the U.S. Air Force Research Laboratory. Chamber 6 is a $1.8 \times 3.0 \mathrm{~m}$ cylindrical, stainless steel vacuum chamber that is evacuated by one dual-stage cryopump and four single-stage cryopanels. During thruster operation, the chamber pressure stabilized at approximately $6.1 \times 10^{-6}$ torr for single thruster operation and $2.3 \times 10^{-5}$ torr for four-thruster operation. Both reported pressures are corrected for xenon. Excessive chamber pressure can adversely influence performance and plume measurements due to entrainment of background neutrals into the thruster discharge chamber and an artificial increase in the electron-neutral collision rate in the plume. In the experiments reported here, the ratio of ingested background mass flux to injected propellant mass flux was determined to be less than $0.5 \%$, and no attempt was made to correct any of the collected data for the effects of chamber pressure. ${ }^{6}$

Table 1 Typical operating conditions for the BHT-200 Hall thruster

\begin{tabular}{lc}
\hline \hline Parameter & Value \\
\hline Discharge voltage, V & $250 \pm 0.5$ \\
Discharge current, A & $0.80 \pm 0.03$ \\
Cathode potential, V & $-8.5 \pm 1.0$ \\
Electromagnet current, A & $1.0 \pm 0.03$ \\
Keeper current, A & $0.5 \pm 0.05$ \\
Keeper voltage, V & $13 \pm 1$ \\
Anode mass flow rate, $\mathrm{sccm}^{\mathrm{a}}$ & $8.5 \pm 0.85$ \\
Cathode mass flow rate, $\mathrm{sccm}^{\mathrm{a}}$ & $1.0 \pm 0.1$ \\
\hline \hline
\end{tabular}

${ }^{a}$ Standard cubic centimeter per minute.

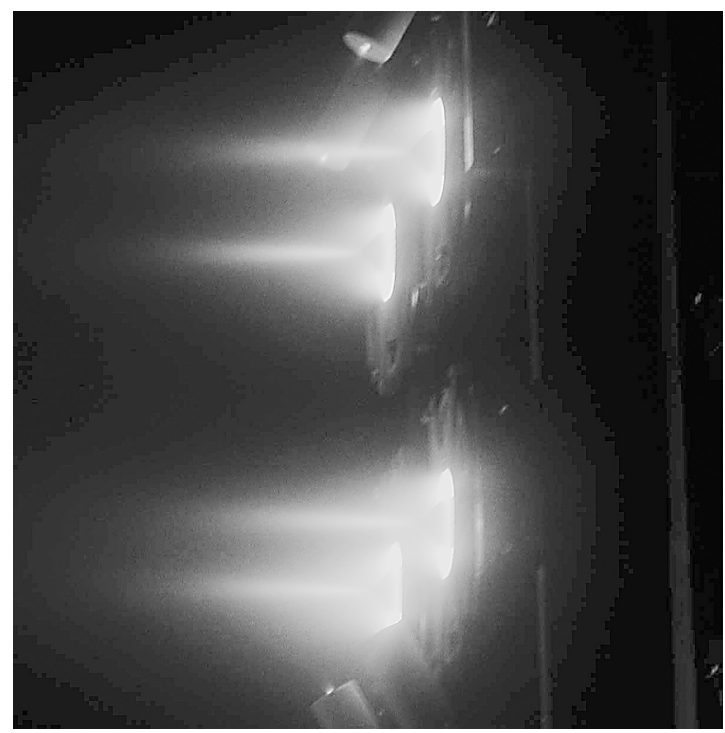

Fig. 1 Low-power Hall thruster cluster in operation.

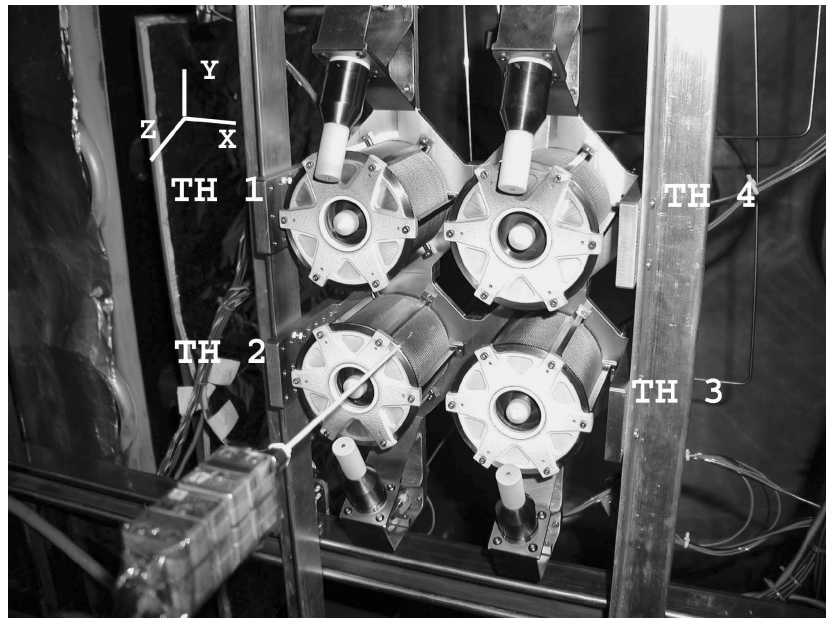

Fig. 2 Thruster naming convention and coordinate system.

\section{Coordinate System}

The naming convention and coordinate system used throughout this experiment are shown in Fig. 2. As shown, the thrusters were labeled as TH 1-4 beginning in the upper left-hand corner and proceeding counterclockwise. The origin of the coordinate system was defined as the midpoint of the cluster in the displayed $X-Y$ plane. The $Z$ coordinate measured the distance downstream of the thruster exit plane. A three-dimensional positioning system was used to sweep probes through the plasma plume.

\section{Triple Probe}

The symmetric triple probe, originally developed by Chen and Sekiguchi, ${ }^{8}$ is a convenient plasma diagnostic for collecting large amounts of data due to the elimination of the voltage sweep required by other electrostatic probes. Additionally, because the probe as a whole floats, the disturbance to the ambient plasma is minimized compared to single langmuir probes, which draw a net current from the discharge. The triple langmuir probe used for these experiments consisted of three tungsten electrodes insulated from each other by an alumina rod. Each electrode was $0.5 \mathrm{~mm}(0.020 \mathrm{in}$.) in diameter and $5.0 \mathrm{~mm}(0.20 \mathrm{in}$.) long. The spacing between the centerlines of adjacent electrodes was approximately $2 \mathrm{~mm}$. The probe was sized to criteria that allowed the standard thin sheath assumptions of probe theory to be applied. ${ }^{9}$ These criteria, which are discussed elsewhere, ${ }^{6}$ are necessary to ensure proper operation of the probe.

Three distinct methods were evaluated to calculate the electron number density and electron temperature from raw triple-probe data. The first is the original method derived by Chen and Sekiguchi ${ }^{8}$ which assumes that the thickness of the sheath surrounding each electrode is negligible compared to the radius of the probe and that each electrode collects an equal ion saturation current. With these assumptions, the plasma density and electron temperature can be deduced using ${ }^{8}$

$$
\begin{gathered}
n=\left(\frac{e m_{i}}{k_{b} T_{e}}\right)^{\frac{1}{2}} \frac{I \exp \left(\frac{1}{2}\right)}{A e^{\frac{3}{2}}\left[\exp \left(e V_{d 2} / k_{b} T_{e}\right)-1\right]} \\
\frac{1-\exp \left(-e V_{d 2} / k_{b} T_{e}\right)}{1-\exp \left(-e V_{d 3} / k_{b} T_{e}\right)}=\frac{1}{2}
\end{gathered}
$$

The second method follows the mathematically rigorous derivation employed by Tilley et al., ${ }^{10}$ which takes into account the slight variations in ion current collected by the three electrodes as a result of their differing voltages. ${ }^{11}$ Unfortunately, the Peterson-Talbot method $^{11}$ used to calculate the current to each electrode requires knowledge of the ion to electron temperature ratio, $T_{i} / T_{e}$. Because this ratio is unknown in most practical situations, it is usually assumed to be constant throughout the plume and to have a value somewhere between 0 and 1 . Although this assumption may be justified in many situations, it is not supported by previously published 
laser-induced fluorescence measurements taken downstream of a Hall thruster, which showed the ion temperature in the plume to remain nearly constant over a large area, whereas the electron temperature varied considerably over a comparable region. ${ }^{12,13}$ When the ambiguity associated with the changing temperature ratio was considered, this method was determined to be of varying validity over the sampled plume region and, therefore, unsuitable for determining the electron temperature and number density profiles.

The third method is very similar to the first, except that the physical probe area shown in Eq. (1) is replaced with an effective collection area, which is defined as the surface area of the sheath surrounding each electrode. An estimate of the sheath thickness is given by the following equation and is approximately five Debye lengths for a xenon plasma ${ }^{14}$ :

$\delta=1.02 \lambda_{D}\left\{\left[\frac{1}{2} \ln \left(m_{i} / m_{e}\right)\right]^{\frac{1}{2}}-(1 / \sqrt{2})\right\}^{\frac{1}{2}}\left\{\left[\frac{1}{2} \ln \left(m_{i} / m_{e}\right)\right]^{\frac{1}{2}}+\sqrt{2}\right\}$

For cylindrical electrodes, such as the ones comprising the triple probe, the sheath area is then given by

$$
A_{S}=A\left[1+\left(\delta / r_{p}\right)\right]
$$

The preceding relations allow the calculation method to proceed by first estimating the density according to Eq. (1) and using this estimate to calculate a value of the sheath thickness according to Eq. (3). The updated collection area from Eq. (4) is then used to determine an updated value of the density, and the procedure continues iteratively until the solution converges. This method is the one used throughout the remainder of this work and is preferred over the other two for several reasons. Unlike the procedure that depends on assuming an ion to electron temperature ratio, the selected method is believed to be of essentially constant validity over the entire plume region because it accounts for changing plasma conditions by explicitly taking into account the effect of the variable Debye length on the derived parameters. Additionally, this method eliminates the slight overprediction of plasma density caused by neglecting the sheath thickness in the collection area of Eq. (1). Various error analyses indicate that the uncertainty in the calculated electron temperature and number density are generally less than 30 and $60 \%$, respectively. ${ }^{8,15}$ The relative uncertainty between multiple data points recorded using the same probe is believed to be significantly lower than the absolute uncertainty because many sources of error, such as asymmetry and uncertainty in the dimensions of each electrode, remain constant from point to point.

\section{Emissive Probe}

Plasma potential measurements were conducted using a floating emissive probe similar to the one described by Haas and Gallimore. ${ }^{16}$ The emitting portion of the probe consisted of a $0.127-\mathrm{mm}-(0.005-$ in.-) diam tungsten filament loop, the ends of which were inserted into double-bore alumina tubing along with $0.508-\mathrm{mm}-(0.020$-in.-) diam molybdenum wire leads. Short lengths of tungsten wire were inserted into the alumina tube to ensure contact between the emitting filament and molybdenum leads. The diameter of the emitting filament loop was approximately $3 \mathrm{~mm}$. Figure 3 shows the emissive probe.

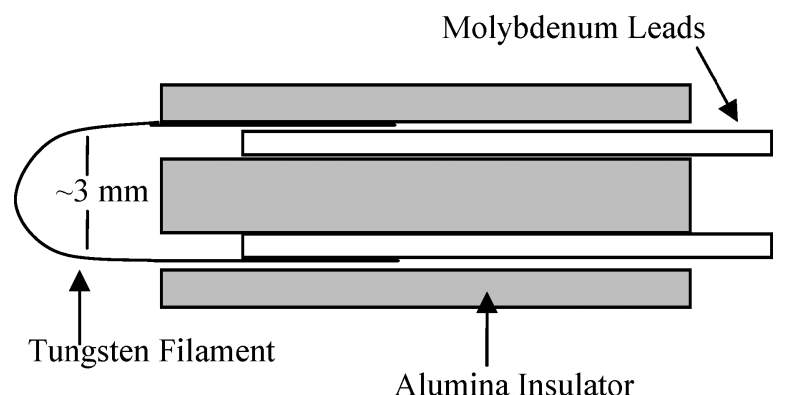

Fig. 3 Emissive probe.
The theory of the emissive probe is well established and results in the conclusion that a thermionically emitting filament will assume the local plasma potential when its emitted electron current is sufficient to neutralize the plasma sheath. ${ }^{17}$ For this experiment, the current necessary to heat the probe was provided by a programmable power supply with floating outputs. At each location in the plume, the current was steadily increased and the potential with respect to ground at the negative terminal of the supply was recorded. This method allowed for verification of a well-defined plateau in the voltage-current trace, which indicated plasma sheath neutralization. When it is considered that the voltage drop across the emitting filament never exceeded $6 \mathrm{~V}$ and that the potential measurements were obtained at the negative side of the probe, the absolute uncertainty in the plasma potential measurements was conservatively estimated to be $+6,-3 \mathrm{~V}$.

\section{Gaussmeter}

The magnetic field downstream of the cluster was mapped using an FW Bell Model 7030 three-axis gaussmeter, which provided a measurement uncertainty of $\pm 0.05 \%$ according to specifications provided by the manufacturer. All measurements were recorded without the thrusters in operation. Although recent work has shown the magnetic field profiles inside an operating Hall thruster to deviate from the applied profiles due to fields induced by the azimuthal electron drift, ${ }^{18}$ the difference is expected to be negligible for the low-power thrusters studied here because of the low-current levels involved. The magnetic field profiles presented in this paper are, therefore, believed to be realistic representations of those that occur downstream of an operational cluster.

\section{Results and Discussion}

\section{Magnetic Field}

Figure 4 shows magnetic field data recorded in the $X Z$ plane of thrusters 2 and 3 and in the $Y Z$ plane of thrusters 3 and 4. In Fig. 4,

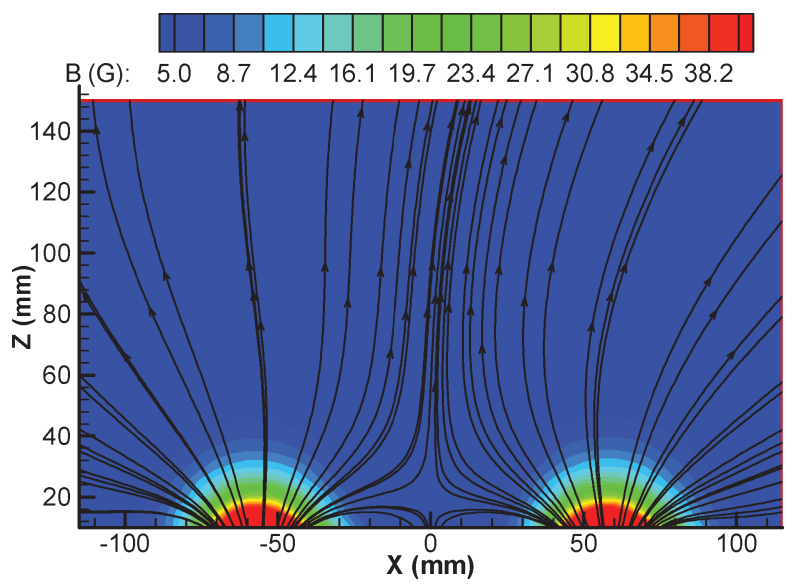

a)

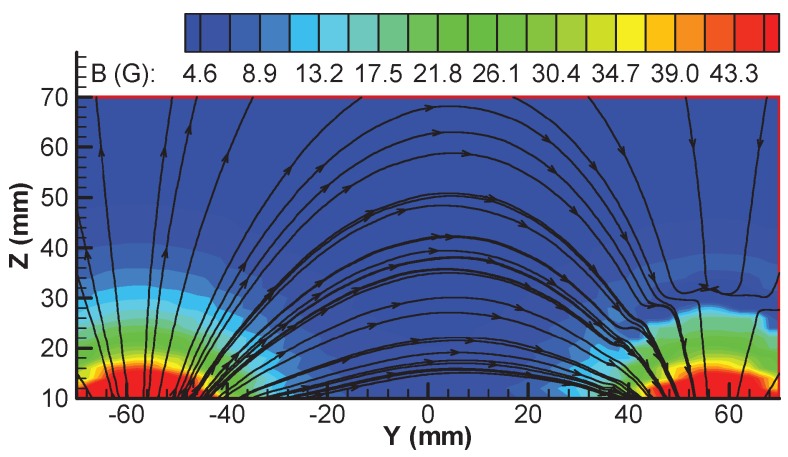

b)

Fig. 4 Magnetic field profiles downstream of a) thrusters 2 and 3 and b) thrusters 3 and 4 . 
the color contours depict total magnetic flux density (field strength), whereas the black lines represent lines of force (vector directions). The differences in Figs. 4 are attributable to the different direction of magnet current flow between thrusters 2 and 4 . Thrusters 2 and 3 were operated with the electromagnets in the nominal configuration, whereas the current flow was reversed in thruster 4. Reversing the polarity of electromagnets in alternate thrusters of a cluster has been suggested as a means of canceling the disturbance torques that typically result from the slight $E \times B$ drift of the beam ions. ${ }^{1,2,19}$ In the work reported here, reversing the polarity of one electromagnet provided a convenient means of assessing the influence of the magnetic field on the plasma potential in the plume, as discussed later.

\section{Plasma Density}

A triple langmuir probe was used to measure the plasma number density at 5-mm intervals in the cluster plume. Data were recorded in both the $X Z$ plane of thrusters 2 and 3 and in the $Y Z$ plane of thrusters 3 and 4 . For both planes, data were recorded with each thruster operating alone and with two thrusters operating simultaneously. Because of the good agreement between the two data sets, only the data recorded in the $Y Z$ plane of thrusters 3 and 4 are reported here.

The plasma density profiles downstream of thrusters 3 and 4 are shown in Fig. 5. As Fig. 5 shows, the maximum number density $50 \mathrm{~mm}$ downstream of the cluster exit plane was roughly $1 \times 10^{18} \mathrm{~m}^{-3}$. This value decreased rapidly in the downstream direction, and by $Z=250 \mathrm{~mm}$, the maximum plasma density decreased by more than an order of magnitude to about $3 \times 10^{16} \mathrm{~m}^{-3}$. Figure 5 shows a well-defined jet structure downstream of each individual thruster. By about $250 \mathrm{~mm}$ downstream, the plumes merged to the point that the density was nearly constant across the width of the cluster and resembled the profile that would be expected downstream of a large monolithic thruster.

Figure 6 shows plasma density profiles at axial distances of 50, 150 , and $250 \mathrm{~mm}$ downstream of the cluster exit plane. The heavy lines in Fig. 6 were obtained by linear superposition of the data recorded with thruster 3 and thruster 4 running independently. The measurements of plasma density taken with both thrusters operating simultaneously agree with the values calculated by superposition to well within the margin of error of the triple-probe diagnostic. This implies that the density in a cluster plume can be predicted by summing the contributions of each individual thruster, as

$$
n=\sum_{j} n_{j}
$$

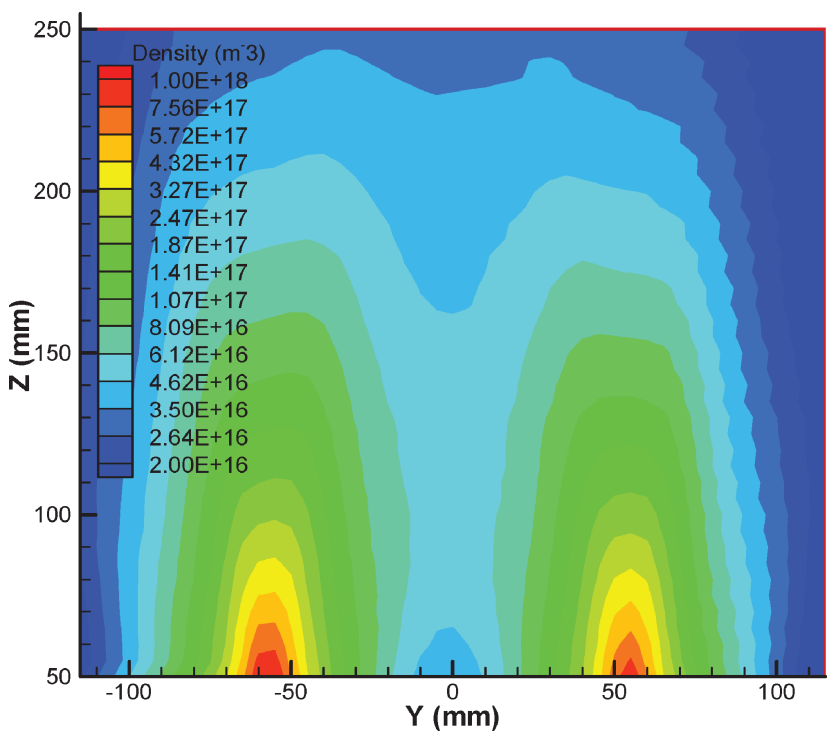

Fig. 5 Plasma density measured downstream of thrusters 3 and 4 using the triple-probe diagnostic.

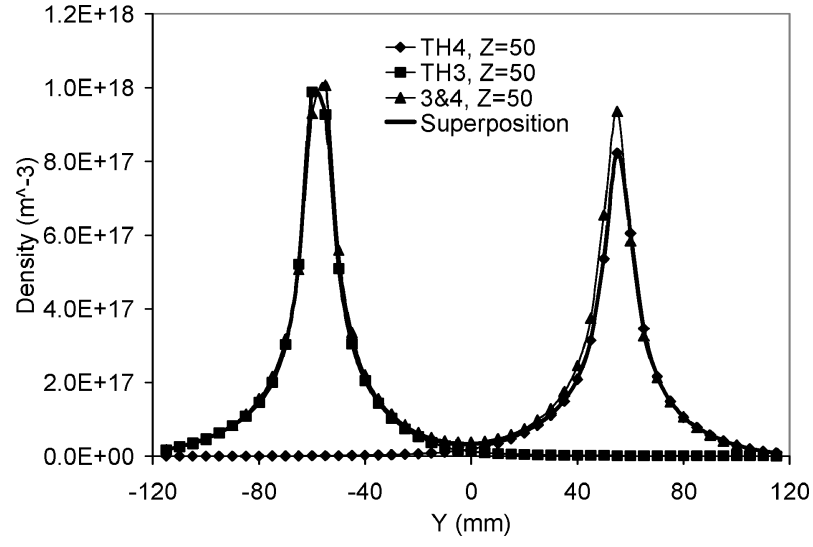

a)

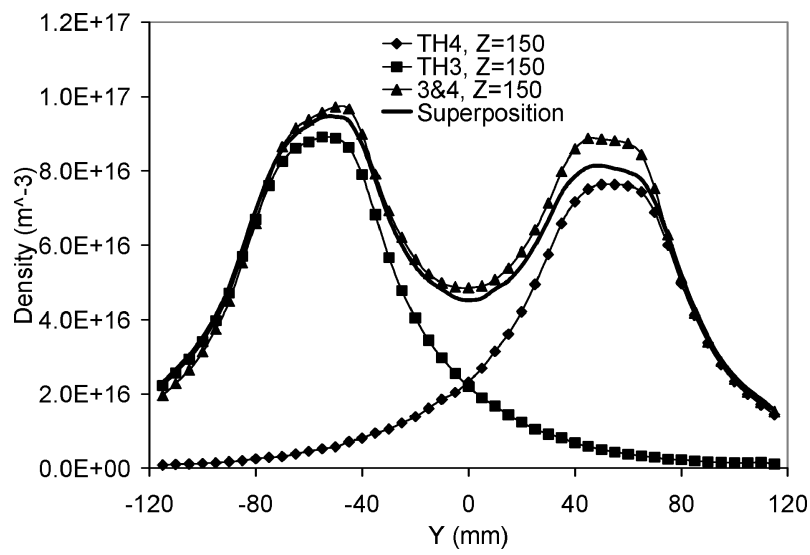

b)

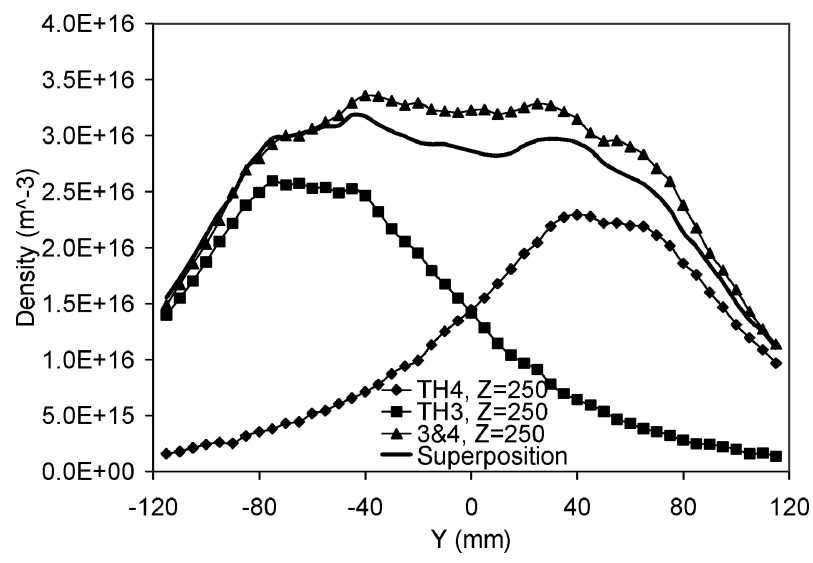

c)

Fig. 6 Plasma density downstream of thrusters 3 and 4: a) $50 \mathrm{~mm}$, b) $150 \mathrm{~mm}$, and c) $250 \mathrm{~mm}$.

\section{Electron Temperature}

The electron temperature contours recorded downstream of thrusters 3 and 4 are shown in Fig. 7. The temperature varied between roughly $3 \mathrm{eV}$ at $Z=50 \mathrm{~mm}$ along the thruster centerlines to less than $1 \mathrm{eV}$ near the boundaries of the sampled region. The data show slight deviations in the electron temperature in the near field when individual thrusters are compared. Measurements recorded downstream of thrusters 2 and 3 (not shown) indicated similar differences; thus, the variations are not believed to be a result of the reversed magnetic field profiles mentioned earlier. Rather, the discrepancies were probably due to tolerances in the manufacturing process or differences in the cumulative time of operation between the devices. The difference in the electron temperature in front of each thruster decreased as a function of downstream distance, and by roughly $Z=90 \mathrm{~mm}$, the difference between the two units was negligible. 


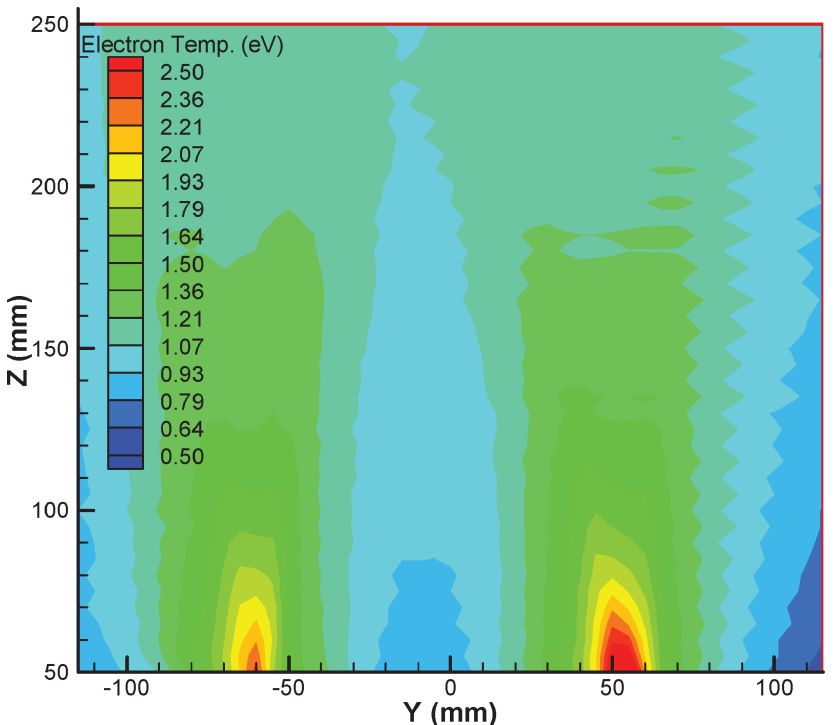

Fig. 7 Electron temperature profile measured downstream of thrusters 3 and 4.

Electron temperature traces measured at axial locations of 50, 150, and $250 \mathrm{~mm}$ are shown in Fig. 8. The method used to predict the electron temperature in the cluster plume from measurements downstream of a single thruster utilizes the equation of state given by Eq. (6). In Eq. (6), $T_{e, 0}$ and $n_{0}$ are reference values taken in the plume of a single thruster and $n$ is the plasma density calculated according to Eq. (5). In Fig. 8, the curves labeled adiabatic were calculated using the reference values measured $50 \mathrm{~mm}$ downstream of thruster 3 along the centerline when it was the only device in operation $\left(n_{0}=9.89 \times 10^{17} \mathrm{~m}^{-3}\right.$ and $\left.T_{e, 0}=2.56 \mathrm{eV}\right)$. As a slight variation on this method, the curves labeled adiabatic (sliding reference) in Fig. 8 were calculated by allowing $T_{e, 0}$ and $n_{0}$ to vary with downstream distance. In this case, the values of density and electron temperature measured along the centerline of thruster 3 at each downstream distance, that is, $Z=50,150$, and $250 \mathrm{~mm}$ in Fig. 8, were used as reference values for predicting cluster properties at the corresponding locations in the cluster plume. In all cases, the ratio of specific heats $\gamma$ was taken to be 1.3 because this value has been shown to provide an excellent fit to experimental data for the plume of a single BHT-200 Hall thruster (see Ref. 6). As shown in Fig. 8, electron temperature values calculated using the following equation agree with the measured data to within the uncertainty of the diagnostic:

$$
T_{e}=T_{e, 0}\left(n / n_{0}\right)^{\gamma-1}
$$

\section{Plasma Potential}

An emissive probe was used to measure the plasma potential at 5-mm intervals in the cluster plume. Results obtained with thrusters 3 and 4 operating simultaneously are shown in Fig. 9. An interesting feature shown in Fig. 9 is the unique plasma potential profile in the area between the thrusters. Between approximately $Y=-30 \mathrm{~mm}$ and $Y=30 \mathrm{~mm}$, the plasma potential increases with downstream distance, indicating that there exists a region where the electric field vector is oriented in the upstream direction. This can be seen clearly in Fig. 10, which shows the plasma potential profiles at various axial locations. The reversed electric field could potentially cause ions produced in the area between the thrusters to be accelerated upstream toward the spacecraft on which the thrusters are mounted. Although this could hypothetically result in an increased erosion rate in some areas due to increased ion impingement, the effect is expected to be negligible because the impinging ions are unlikely to experience accelerating potentials greater than a few volts in the reverse direction. ${ }^{6}$

A widely used method for predicting the potential profiles in a plasma is to relate them to profiles of magnetic field strength. Along a magnetic field line, the motion of electrons is governed purely by

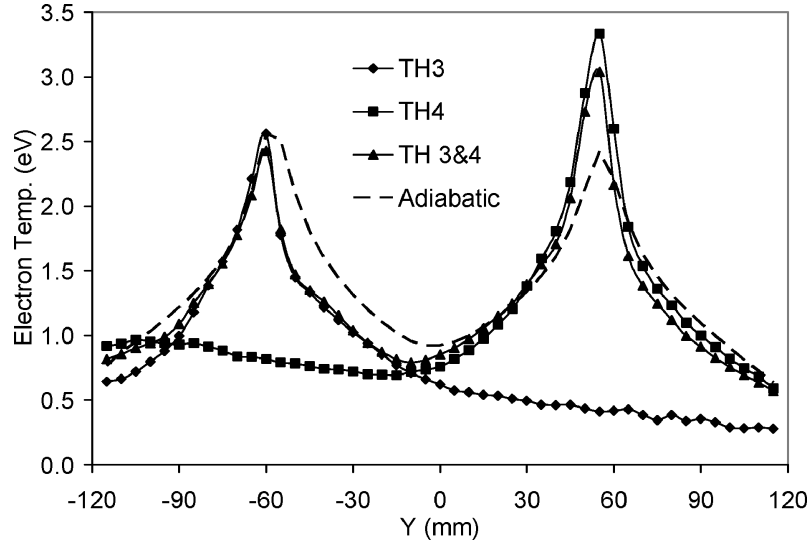

a)

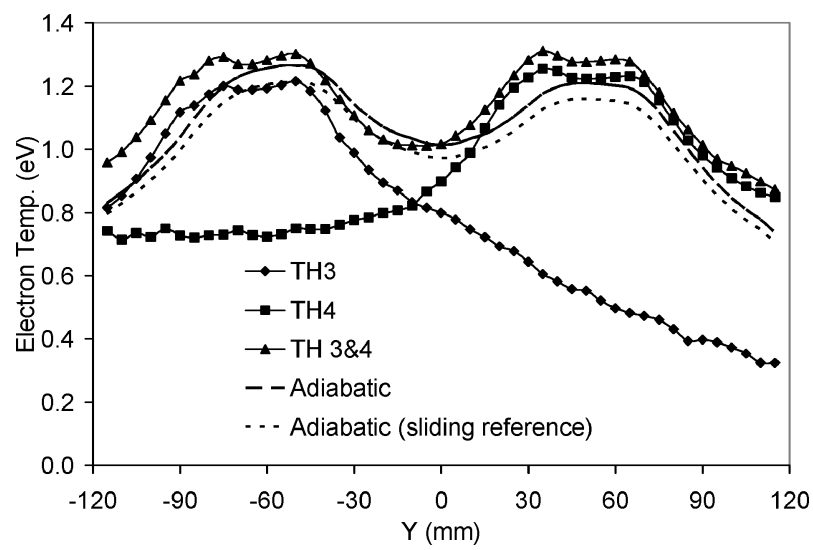

b)

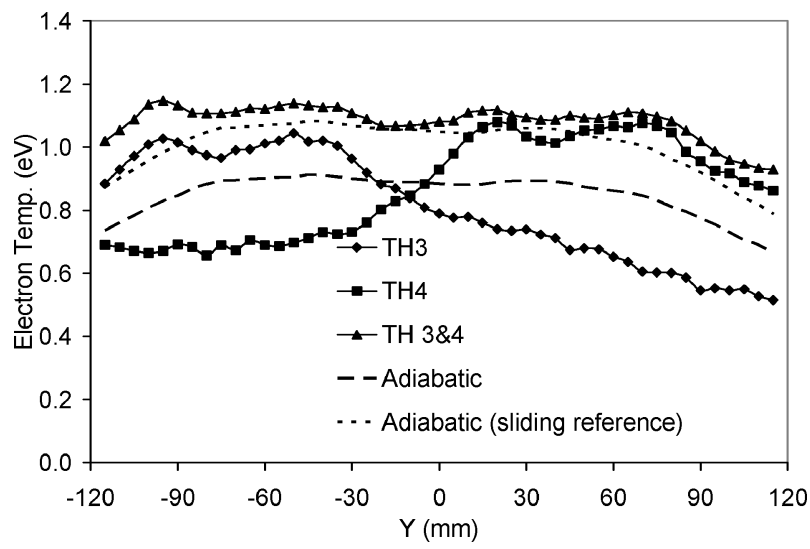

c)

Fig. 8 Electron temperature at a) $Z=50 \mathrm{~mm}$, b) $Z=150 \mathrm{~mm}$, and c) $Z=250 \mathrm{~mm}$.

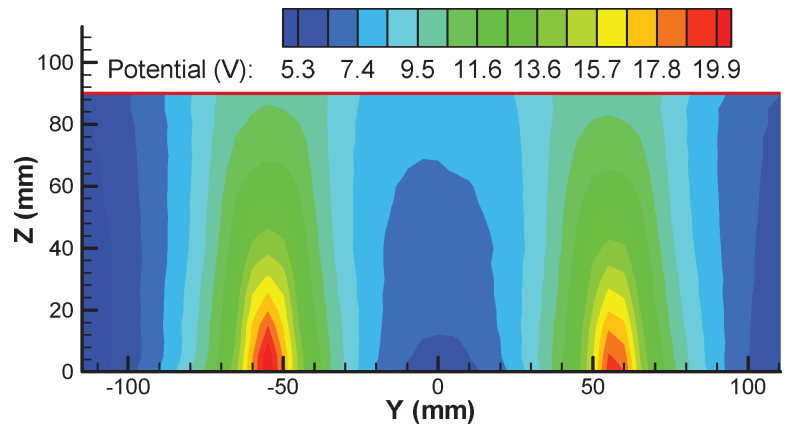

Fig. 9 Plasma potential downstream of thrusters 3 and 4. 


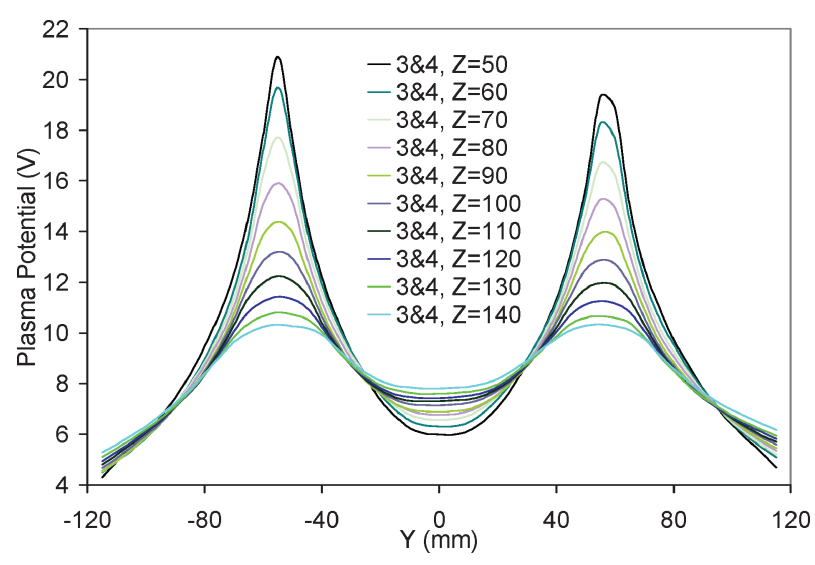

Fig. 10 Evolution of plasma potential downstream of two simultaneously operating Hall thrusters.

electrostatic forces and can be described by the well-known Boltzmann relation (see Ref. 20). This leads naturally to the definition of a thermalized potential, $\phi_{T}$, which is given by Eq. (7) and conserved along a line of force. ${ }^{21}$ In the derivation of the following equation, the electron temperature has been assumed constant along lines of force:

$$
\phi_{T} \equiv \phi-\left(k_{B} T_{e} / e\right) \ln \left(n / n_{0}\right)
$$

The concept of thermalized potential is useful in the design of Hall thrusters because it shows that the magnetic field lines can be approximated as equipotential lines in situations where the electron temperature is negligible compared to the plasma potential. In other words, the thermalized potential is a useful tool for predicting plasma potential in situations where electrons are tightly bound to the magnetic field lines, such as inside the thruster discharge channel. This method, however, is less useful in the thruster plume because the correction term due to thermal effects and density gradients can be as large as the plasma potential.

When the plasma potential data of Fig. 9 are compared to the magnetic field profiles shown in Fig. $4 \mathrm{~b}$, it is clear that the lines of force do not correspond to equipotential contours. Furthermore, plasma potential measurements recorded downstream of thrusters 2 and 3 (not shown) were observed to be in very good agreement with those shown in Fig. 9 for thrusters 3 and 4 despite the marked differences in magnetic field shape shown in Fig. 4 for both cases. The fact that the magnetic field does not appear to control the plasma potential in the thruster plume is not surprising because the field strength is generally less than $10 \mathrm{G}$ and the electrons are only weakly magnetized in the areas of interest.

In the Hall thruster plume, thermal effects and density gradients are dominant over the effects of the magnetic field, and the plasma potential can be described by the Boltzmann relation, which is given by Eq. (8) (see Ref. 6). The profiles calculated using Eq. (8) are shown in Fig. 11 and generally agree to within $1.5 \mathrm{~V}$ of the measured values, except in the most upstream locations of the sampled region. In utilizing Eq. (8), the reference density $\left(n_{0}=5 \times 10^{13} \mathrm{~m}^{-3}\right.$ in this case) was chosen to make the plasma potential calculated along the centerline of thruster 3 at $Z=100 \mathrm{~mm}$ match the measured value. Although the choice to match the value at $100 \mathrm{~mm}$ was arbitrary, a similar approach is expected to be valid in most practical cluster configurations because the data presented here show the plasma potential directly downstream of one thruster to be largely unaffected by the surrounding devices. Implementation of Eq. (8) along with Eqs. (5) and (6), thus, allows the most basic plasma properties downstream of a cluster of identical Hall thrusters to be predicted based solely on measurements or simulations of a single unit. Results obtained in this way appear to be accurate to within the margin of error of typical plasma diagnostics:

$$
\phi=\left(K_{B} T_{e} / e\right) \ln \left(n / n_{0}\right)
$$

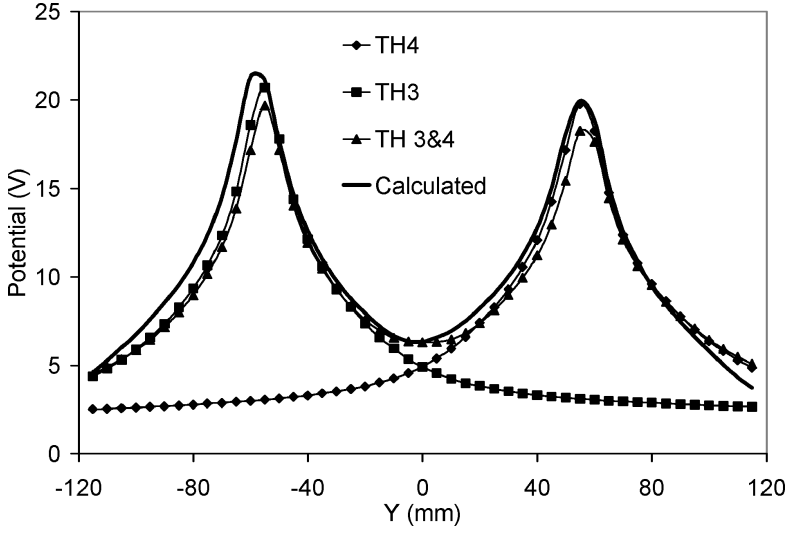

a)

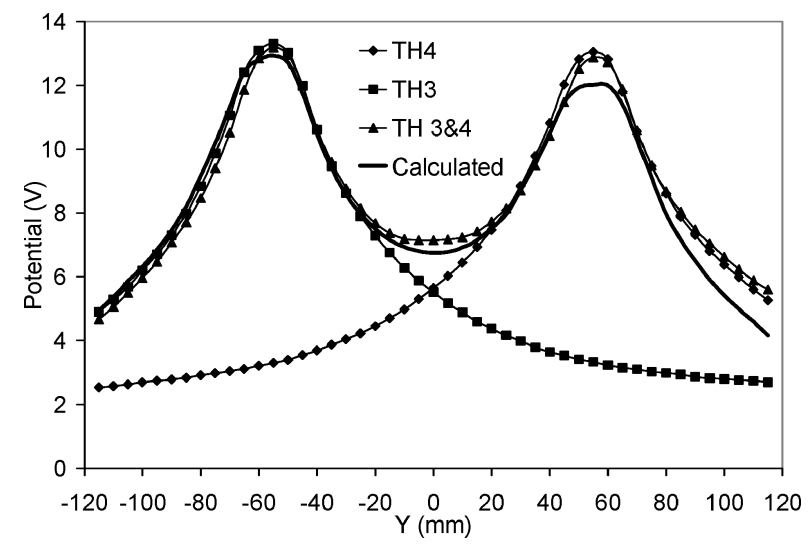

b)

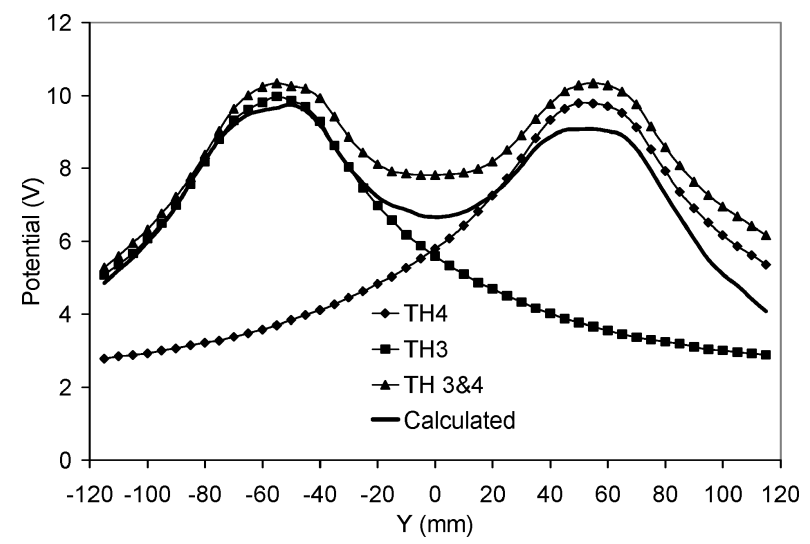

c)

Fig. 11 Plasma potential profiles at a) $Z=60 \mathrm{~mm}$, b) $Z=100 \mathrm{~mm}$, and c) $Z=140 \mathrm{~mm}$.

\section{Conclusions}

Measurements of plasma density, electron temperature, and plasma potential in the plume of a low-power Hall thruster cluster show that these properties can be accurately predicted using simple analytical relations and knowledge of a single thruster plume. In particular, the plasma density can be predicted by simple linear superposition of the contribution from individual devices, whereas the electron temperature can be obtained from an adiabatic equation of state. After predictions of electron number density and electron temperature are obtained, these results can then be used to estimate the resulting plasma potential via the Boltzmann relation. Predictions of plasma properties made using these techniques are accurate to within the margin of error of typical plasma diagnostics.

\section{Acknowledgments}

This work was performed under the auspices of the Air Force Office of Scientific Research Grant F49620-02-1-0051 and 
AFRL-ERC Grant RPO20870. The authors wish to thank Mitat Birkan of the Air Force Office of Scientific Research and David Campbell of ERC, Inc., for sponsoring this research.

\section{References}

${ }^{1}$ Spores, R. A., Spanjers, G. G., Birkan, M., and Lawrence, T. J., "Overview of the USAF Electric Propulsion Program," AIAA Paper 20013225 , July 2001

${ }^{2}$ Spanjers, G. G., Birkan, M., and Lawrence, T. J., "The USAF Electric Propulsion Research Program,” AIAA Paper 2000-3146, July 2000.

${ }^{3}$ Beal, B. E., Gallimore, A. D., and Hargus, W. A., "Preliminary Plume Characterization of a Low-Power Hall Thruster Cluster," AIAA Paper 20024251, July 2002

${ }^{4}$ Hargus, W. A., and Reed, G., "The Air Force Clustered Hall Thruster Program," AIAA Paper 2002-3678, July 2002.

${ }^{5}$ Beal, B. E., and Gallimore, A. D., "Energy Analysis of a Hall Thruster Cluster," Electric Rocket Propulsion Society, IEPC Paper 2003-0035, March 2001.

${ }^{6}$ Beal, B. E., "Clustering of Hall Effect Thrusters for High-Power Electric Propulsion Applications," Ph.D. Dissertation, Dept. of Aerospace Engineering, Univ. of Michigan, Ann Arbor, MI, April 2004.

${ }^{7}$ Hruby, V., Monheiser, J., Pote, B., Rostler, P., Kolencik, J., and Freeman, C., "Development of Low-Power Hall Thrusters," AIAA Paper 99-3534, June 1999

${ }^{8}$ Chen, S., and Sekiguchi, T., "Instantaneous Direct-Display System of Plasma Parameters by Means of Triple Probe," Journal of Applied Physics, Vol. 36, No. 8, 1965, pp. 2363-2375.

${ }^{9}$ Schott, L., "Electrical Probes," Plasma Diagnostics, edited by W. LochteHoltgreven, AIP Press, Woodbury, NY, 1995, pp. 668-731.

${ }^{10}$ Tilley, D. L., Kelly, A. J., and Jahn, R. G., "The Application of the Triple Probe Method to MPD Thruster Plumes," AIAA Paper 90-2667, July 1990.

${ }^{11}$ Peterson, E. W., and Talbot, L., "Collisionless Electrostatic Single-
Probe and Double-Probe Measurements," AIAA Journal, Vol. 8, No. 12, 1970, pp. 2215-2219.

${ }^{12}$ Williams, G. J., Jr., Smith, T. B., Gulczinski, F. S., III, Beal, B. E., Gallimore, A. D., and Drake, R. P., "Laser Induced Fluorescence Measurement of Ion Velocities in the Plume of a Hall Effect Thruster," AIAA Paper 99-2424, June 1999.

${ }^{13}$ Haas, J. M., "Low-Perturbation Interrogation of the Internal and NearField Plasma Structure of a Hall Thruster Using a High-Speed Probe Positioning System," Ph.D. Dissertation, Dept. of Aerospace Engineering, Univ. of Michigan, Ann Arbor, MI, Jan. 2001.

${ }^{14}$ Hutchinson, I. H., Principles of Plasma Diagnostics, Cambridge Univ. Press, New York, 2002, Chap. 3.

${ }^{15}$ Tilley, D. L., Gallimore, A. D., Kelly, A. J., and Jahn, R. G., "The Adverse Effect of Ion Drift Velocity Perpendicular to a Cylindrical Triple Probe," Review of Scientific Instruments, Vol. 65, No. 3, 1994, pp. 678-681.

${ }^{16}$ Haas, J. M., and Gallimore, A. D., "Internal Plasma Potential Profiles in a Laboratory Model Hall Thruster," Physics of Plasmas, Vol. 8, No. 2, 2001, pp. 652-660.

${ }^{17}$ Kemp, R. F., and Sellen, J. M., Jr., "Plasma Potential Measurements by Electron Emissive Probes," Review of Scientific Instruments, Vol. 37, No. 4, 1966, pp. 455-461.

${ }^{18}$ Peterson, P. Y., Gallimore, A. D., and Haas, J. M., "An Experimental Investigation of the Internal Magnetic Field Topography of an Operating Hall Thruster," Physics of Plasmas, Vol. 9, No. 10, 2002, pp. 4354-4362.

${ }^{19}$ Manzella, D. H., "Stationary Plasma Thruster Ion Velocity Distribution," AIAA Paper 94-3141, June 1994.

${ }^{20}$ Keidar, M., and Boyd, I. D., "Effect of a Magnetic Field on the Plasma Plume from Hall Thrusters," Journal of Applied Physics, Vol. 86, No. 9, 1999, pp. 4786-4791.

${ }^{21}$ Morozov, A. I., Esipchuck, Y. V., Tilinin, G. N., Trofimov, A. V., Sharov, Y. A., and Shchepkin, G. Y., "Plasma Accelerator with Closed Electron Drift and Extended Acceleration Zone," Soviet Physics-Technical Physics, Vol. 17, No. 1, 1972, pp. 38-45.

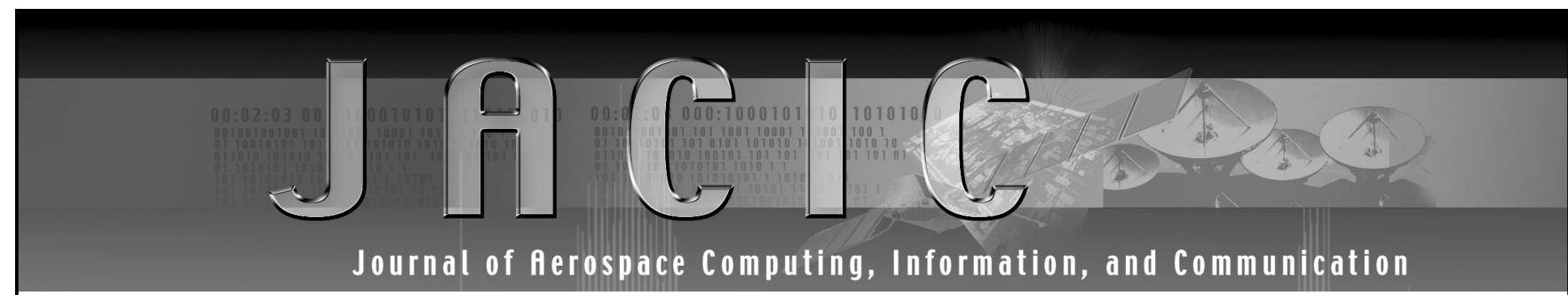

Editor-in-Chief: Lyle N. Long, Pennsylvania State University

AIAA is launching a new professional journal, the Journal of Aerospace Computing, Information, and Communication, to help you keep pace with the remarkable rate of change taking place in aerospace. And it's available in an Internet-based format as timely and interactive as the developments it addresses.

\section{Scope:}

This journal is devoted to the applied science and engineering of aerospace computing, information, and communication. Original archival research papers are sought which include significant scientific and technical knowledge and concepts. The journal publishes qualified papers in areas such as real-time systems, computational techniques, embedded systems, communication systems, networking, software engineering, software reliability, systems engineering, signal processing, data fusion, computer architecture, high-performance computing systems and software, expert systems, sensor systems, intelligent sys- tems, and human-computer interfaces. Articles are sought which demonstrate the application of recent research in computing, information, and communications technology to a wide range of practical aerospace engineering problems.

Individuals: $\$ 40 \cdot$ Institutions: $\$ 380$

$\rightarrow$ To find out more about publishing in or subscribing to this exciting new journal, visit www.aiaa.org/jacic, or e-mail JACIC@aiaa.org.

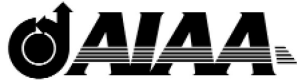

American Institute of Aeronautics and Astronautics 